\title{
Primary dyscalculia after a medial frontal lesion of the left hemisphere
}

\author{
F Lucchelli, E De Renzi
}

\begin{abstract}
A patient had an infarct in the territory of the left anterior cerebral artery, which destroyed the medial cortex of the frontal lobe, and presented with a picture of primary dyscalculia. Lexical and syntactic processing of verbal and arabic numbers and comprehension of operation symbols were intact, but retrieval of basic, overlearned facts was mildly impaired and execution of calculation procedures was more severely impaired. As the same type of procedure could be passed or sometimes failed it suggests a deficit of activation of the appropriate procedures. The location of the lesion was unusual and suggested participation of medial frontal areas in calculation processes.
\end{abstract}

(F Neurol Neurosurg Psychiatry 1993;56:304-307)

An impairment of number processing and calculation skills is not an uncommon manifestation of brain damage, but in the majority of cases it results from the derangement of more general abilities, such as language, attention, memory, space perception, that take place when performing arithmetical tasks. The dyscalculia observed in these patients has been labeled secondary and contrasted with primary dyscalculia, ${ }^{1}$ in which the deficit stands out as particularly severe for other cognitive abilities and suggests the disruption of specific mechanisms. ${ }^{23}$

Dyscalculia has been recently analysed with models that specify the component native of the cognitive mechanisms underlying calculation. ${ }^{45}$ There are two distinct systems: one for number processing and the other for calculation. The former would comprise independent subsystems for comprehension and production of arabic and verbal number form, the latter subsystems for comprehension of arithmetical signs (for example, $\times,+,-$ ), retrieval of arithmetical facts (for example, the multiplication table) and execution of calculation procedures. The model assumes that the input of number processing is converted to an abstract representation, which is used both to activate the calculation system and to trigger appropriate commands for speech and writing centres.

The modular nature of this functional architecture predicts the selective breakdown of its components after brain damage and a search of the literature enabled McCloskey and Caramazza ${ }^{4}$ to collect evidence support- ing several dissociations that agree with the expectations from the model.

The patient we report is unusual for two reasons: 1) Because the case shows a deficit strictly confined to the calculation system, with preservation not only of other cognitive abilities, but also of the number processing system; 2) Because of the unusual site of the lesion, which involved the medial areas of the left frontal lobe.

\section{Case report}

The patient, a 22 year old male, was a third year music student. Before entering the academy of music to study the organ, he had attended liceo classico, the most demanding type of high school in Italy. He did not have difficulty in learning mathematics. In June of 1990 he complained of headache and unsteadiness. Admitted to a neurosurgical department, a cerebellar tumour was diagnosed and on 10 July 1990 he had a grade 2 cystic glioma of the vermis and the right inferior cerebellar peduncle which was completely resected. The post-operative course was uneventful until 23 July 1990, when he complained of a severe headache and a right lower limb weakness. He subsequently became mute and presented with a complete paresis of the right lower limb. When we first saw the patient on 9 August 1990, he showed the syndrome of crural and proximal arm paresis typical of anterior cerebral artery infarction. Grasping, but not groping, was present in both hands. His behaviour was abnormal. $\mathrm{He}$ spontaneously kept his eyes closed and did not initiate talking. He gave correct answers to simple questions but with no more than two words. With more complex questions (for example, "tell me how Jesus Christ died") he remained silent. Visual naming was in the normal range (54/60) according to a test standardised on 70 normal controls. " $\mathrm{He}$ gave only five names of animals in one minute and four names on three phonetic cues $(P, F, L)$, with one minute for each.

On 24 August 1990, an MRI confirmed the presence of an infarct involving the whole territory of the left anterior cerebral artery (figure). The lesion encroached upon areas $10,11,12,24,23$ and the medial extension of area 6 and $4 .^{6}$ The anterior half of the corpus callosum was also damaged.

No cause for the cerebrovascular accident was identified by neurosurgeons.

The patient progressively improved and by September his language had recovered,
Received 24 February 199 and in revised form 1 May 1992.

Accepted 14 May 1992 

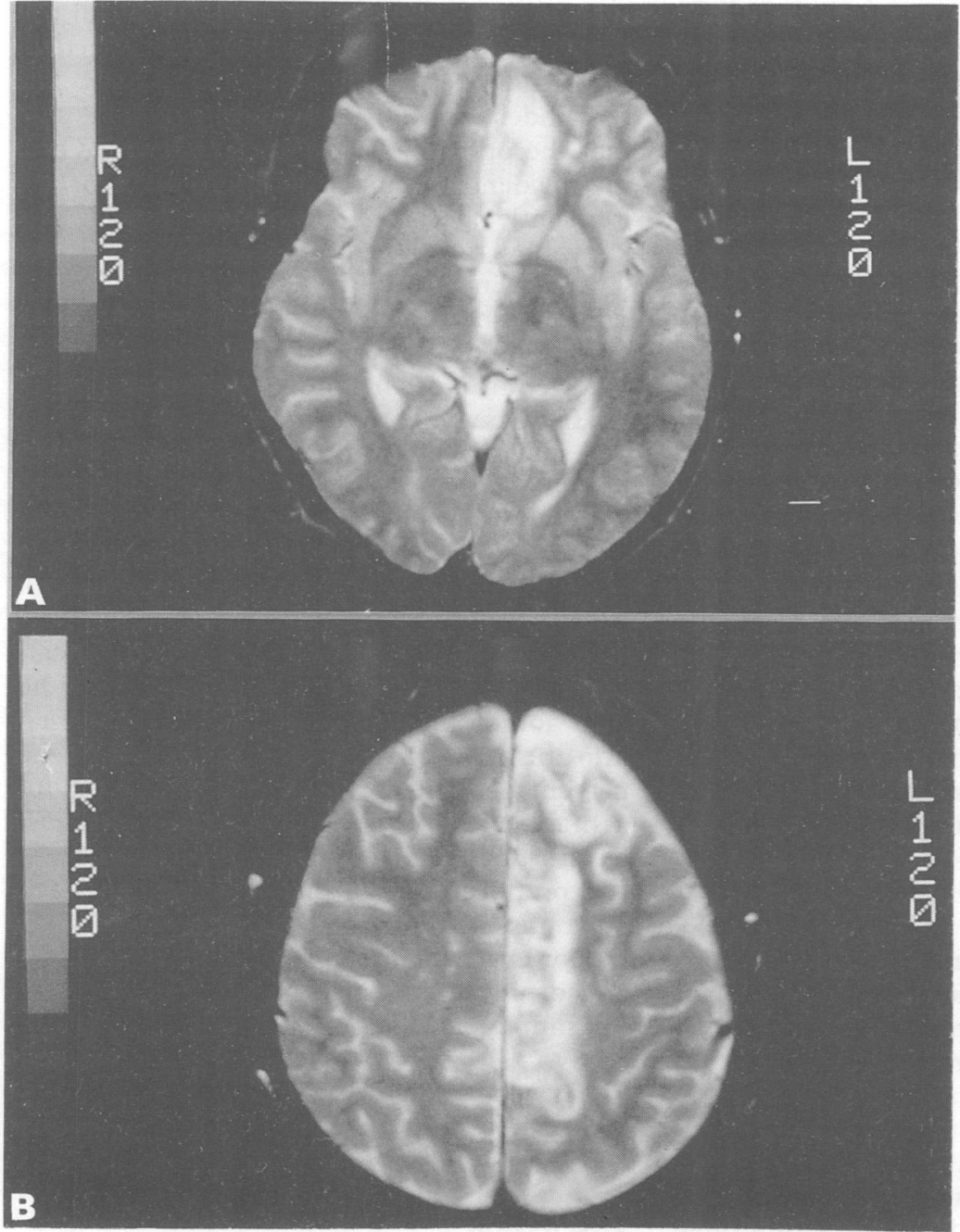

Extent of the MRI lesion. $A$ and B: axial slices.

though he remained somewhat limited in spontaneous speech. During this period a difficulty with calculation was noted by the patient which prompted the investigations that were carried out from September to November 1990.

Most of the tests were carried out in September 1990, and those that did not yield scores in the normal range were repeated in October or November 1990.

The WAIS FS IQ was 98 in September and 111 in October, with an improvement of more than 20 points on the PIQ (from 82 to 104) and a largely stable VIQ (from 110 to 114). On the arithmetic subtest the patient had a weighted score of 8 in both sessions with failure on the last, more complex items. His intellectual proficiency was confirmed by the scores achieved on the Raven Progressive Matrices, series A, B, C, D (43/48), Wisconsin Card Sorting Test (6 categories of 6) and revised Wegl test (9 of 15). ${ }^{7}$

The patient was given: a) Verbal learning tests (story recall, paired associates, 10-word list); b) Visual memory tests (Rey figure, recurring face recognition); and c) Spatial memory tests (visual stepping stone maze). On all tests he performed in the normal range.

No deficit of oral and written language was apparent on a standardised aphasia battery. He scored $32 / 36$ on the Token Test and $72 / 85$ on the Boston Naming Test. $\mathrm{He}$ was also able to read musical scores and the ancient Greek tragedy Medea, which he knew from his classical studies, with correct metrics.

Up to the middle of October the patient occasionally presented the alien hand symptom, with the right hand carrying out performances that interfered with his activity, for example, when turning the pages of a book he was reading. The symptom then subsided. On a limb movement imitation test he scored $70 / 72$ with the right hand and $68 / 72$ with the left hand (cut-off score 62/72), but on a test requiring him to mime the use of single objects, his performance with either hand was awkward and inaccurate, though the general configuration of the movement was correct. His copy of the Rey figure was flawless (36/36).

Arithmetic performance Processing of verbal and arabic numbers was rapid and correct, for both comprehension and production. $\mathrm{He}$ read and wrote numbers up to 7 digits without error and hesitation, easily pointed to the larger number in a pair and quickly gave a correct estimation of a random array of 2-100 black dots ( 2 seconds exposure) in 60 trials. $^{8}$ $\mathrm{He}$ had no difficulty in transcoding Arabic numbers to written verbal numbers and vice versa.

He named and understood without difficulty the meaning of operation symbols $(t$, $-, \times)$, responded correctly to 10 general knowledge questions concerning numerical facts (such as, days of the week, pairs in a dozen,) and gave reasonably correct answers to 10 numerical estimation questions (such as, the height of an average Italian woman). ${ }^{8}$

Oral calculations His impairment in oral calculation became apparent when he was requested to retrieve basic arithmetic facts, that is, facts that are learnt from memory by virtually every educated adult, such as those involved in multiplication tables and in addition and subtraction of digits from 1 to 19 .

Addition On thirty seven trials requiring him to add combinations of numbers from 1 to 9 (such as, $5+3$ ), he produced two errors and seven delayed correct responses (more than 2 seconds).

His ability to add number combinations of 1 to 9 to number combinations of 11 to 19 was tested giving the larger of the two numbers either first (such as, $15+3$ ) or second (such as, $8+12$ ). Only responses given under 5 seconds and without self-corrections were accepted as correct. There was no difference between the two conditions $(83 \%$ correct responses in the former and $82 \%$ in the latter) and they were combined for a total of 144 trials, which produced a $6 \%$ error rate, with $3 \%$ self-corrected errors and $9 \%$ delayed correct responses (more than 5 seconds). When numbers from 11 to 19 were added to numbers from 11 to 19 (such as $15+13$ ) the rate of correct responses decreased to $68 \%$. In 110 trials there was an $18 \%$ error rate, 
with $9 \%$ self-corrected errors and $5 \%$ delayed correct responses.

Ten trials requiring the addition of two digit numbers, both larger than 19 , yielded 5 errors and two correct responses given after 30 seconds.

\section{Subtraction}

Thirty six subtractions of single digit numbers were all correct, though in two cases delayed ( $>2$ seconds). Seventy two attempts to subtract a single digit from a two digit number, (for example, 11 to 19), produced $78 \%$ correct responses, $4 \%$ errors, $3 \%$ selfcorrected errors and $15 \%$ delayed responses (more than 5 seconds).

Forty five trials in which a two digit number is subtracted from a two digit number, all comprised between 10 and 19 , produced $91 \%$ correct responses, $4 \%$ errors, $2 \%$ selfcorrected errors and $3 \%$ delayed responses (more than 5 seconds). When, the larger number was from 20 to 99 , however, there were only $40 \%$ correct responses, $30 \%$ errors and $30 \%$ delayed responses (more than 5 seconds) of 10 trials.

\section{Multiplication}

Multiplying all number combinations between 2 and 9 yielded $84 \%$ correct responses, $8 \%$ delayed responses (more than 5 seconds), $5 \%$ self-corrected errors and 3\% errors.

\section{Division}

He was given 30 division calculations, 10 with a two digit dividend and 20 with a three digit dividend. The divisor always had one digit and all divisions were without remainder. Only responses given in 15 seconds were accepted. He made 15 errors $(50 \%)$.

Two normal controls, matched for sex, age and years of education, performed the above oral calculations accurately and rapidly.

\section{Written calculations}

The errors made in written calculations varied according to the type of operation. On addition, only errors of facts were observed (for example, $83+15=99$ ). When subtracting, errors of borrowing were by far the most frequent. Errors of addition and of carrying were the main cause of faulty multiplications, but in one instance a complete misconception of the overall operation schema was noticed: in performing $38 \times 4$, the patient wrote down separately the products of $8 \times 4$ and 3 $\times 4$ and then added them up. Errors pointing to a severe disruption of procedure became more frequent with divisions. Sometimes there was a complete block when confronted by elementary divisions, such as 6 $\div 4$, where he commented: "It strikes me that I ought to write 0 ," but actually wrote nothing. Requested to divide 450 by 3 , he took the first two figures together (45) and wrote 9 as the quotient. Having to divide 36 by 41 , he correctly wrote 0 at the quotient, then subtracted 36 from 41 , wrote 5 , lowered 0 and divided 50 by 41 . A common error occurred when, having underestimated the number of times the divisor was contained in the dividend, he failed to realise that the remainder was larger than the divisor and that the operation had to be checked. When the dividend was smaller than the divisor, he frequently became confused and did not know how to proceed.

Errors increased on more complex operations. He had forgotten the rule for carrying out multiplication and division of numbers followed by a decimal point.

He was unable to mentally calculate simple percentages (for example, $50 \%$ of 10 , or $20 \%$ of 100). When the calculation was carried out with written numbers, he was no longer able to recall the correct procedure: such as, computing $20 \%$ of 50 when he divided 50 by 20 . After a number of trials, he spontaneously remembered the method and was able to calculate $70 \%$ of 200 and $17 \%$ of 3400 , but he later forgot the method again and relapsed into the error of dividing the number by the percentage. The conversion of a fraction into a number (such as, 1/5 =0.20) was almost always incorrect when calculated mentally and produced serious difficulty in written calculations.

However, the concept of the square root and of power was preserved and using them for simple calculations were carried out correctly.

The patient was seen again in November 1991 and found to be unchanged.

\section{Discussion}

The present case adds to the understanding of acalculia in two ways: 1) It provides an example of a disorder strictly confined to the cognitive systems for calculation, and 2) Its lesion involves anatomical structures of the left hemisphere that have not been previously implicated in the mechanisms of calculation deficits.

At the time of testing, the neurological and neuropsychological symptoms caused by the left anterior cerebral artery infarct had cleared almost completely, leaving the patient free from language, praxis, visuoperceptual and visuo-spatial impairments. Intellectual abilities were well within normal limits and no deficits were shown by tests considered sensitive to frontal lobe damage. Verbal, visual and spatial memory was intact. Thus dyscalculia was an isolated area of cognitive deficit, independent of the impairment of other mental abilities and demonstrated the disruption of a specific system subserving arithmetical processes. Its impairment concerned the two major components of the calculation system, retrieval of basic arithmetical facts and execution of calculation procedures. ${ }^{9}$ However, it left the mechanisms intact for number processing and the ability to comprehend operational symbols.

The difficulty in remembering arithmetical information was minor, mainly resulting in the lengthening of the time taken to make a correct response or in self-corrected errors. It 
must be stressed that arithmetical facts are highly automatic in a person with the educational background of our patient.

What was more severe and independent of number facts impairment were the errors attributable to a faulty retrieval of calculation procedures. They can be classified under two headings, those associated with the inability to remember "carry" and "borrow" procedures, and those reflecting a more profound misconception of the nature of the requested operation. The former, which are sometimes also observed in normal subjects, is likely to be dependent on the attentional load demanded by a dual task, in which an element of the previous step of the operation must be kept in mind while focusing on the next one. The latter, which occurred in more complex operations, caused a momentary block in retrieving the pertinent procedure and resulted either in a state of bewilderment and inability to proceed, or in the application of rules inappropriate for that operation. For none of the operations tested, including percentages, fractions and powers, was there evidence of an irreparable loss of ability, or for a consistent pattern of errors, and his erratic performance can be best interpreted as depending on the faltering activation of the calculation functional system.

The lesion responsible for the symptoms was unusual, since no previous case of dyscalculia has been reported following damage to the medial regions of the left frontal lobe. There is consensus in the literature that the area crucially involved in the production of primary acalculia (namely, in the absence of spatial and language deficits) is centred around the temporo-parietal-occipital cortex on the lateral surface of the left hemisphere. ${ }^{10}$ No evidence of damage to this region was shown by MRI. The relation that the frontal locus of lesion bore to dyscalculia can only be a matter of speculation. It is known that injury to the medial frontal cortex and cingulate gyrus can produce, especially if bilateral, a state of akinesia and mutism, of which our patient gave clear evidence in the early stage of his disease. This was no longer apparent when dyscalculia was investigated. There was no evidence of an inability to initiate mental processes, even when the patient was engaged in difficult intellectual tasks, certainly equally or more demanding in terms of attentional resources than the retrieval of numerical facts. An alternative hypothesis is that the left medial frontal lobe plays a direct role in the retrieval of calculation procedures, but that its autonomous contribution has escaped the attention of neuroscientists as it has never been formally tested. Oral calculation is listed among the tasks given to 10 consecutive patients with left anterior cerebral artery infarct ${ }^{11}{ }^{12}$, but unfortunately monitoring was not made of their performance. The assessment of computation may be difficult in patients who show an inhibition of their ability to give verbal responses, as is frequently the case in the early stage of a left anterior cerebral artery infarct.

This study implies that careful investigation of calculation processes may be useful in patients with medial frontal lesion.

This research was supported by grants from CNR and MPI to Dr E. De Renzi. We are grateful to Dr H Buchtel for his comments on an earlier version of the paper.

1 Berger $\mathrm{H}$ Ueber Rechenstoerungen bei Herderkrankungen des Grosshirns. Arch $f$ Psychiat $U$ Nervenkr 1926;78: 238-67.

2 Boller F, Grafman J. Acalculia: historical development and current significance. Brain and Cogn 1983;2 205-23.

3 Hécaen $\mathrm{H}$, Angelergues $\mathrm{R}$, Houilier S. Les variétés cliniques des acalculies au cours des lésions rétrorolandiques. Approche statistique du probléme. Rev Neurol 1961;105:85-103

4 McCloskey M, Caramazza A. Cognitive mechanisms in number processing and calculation: evidence from dyscalculia. Brain and Cogn 1985;4:171-196.

5 McCloskey M, Sokol SM, Goodman RA. Cognitive processes in verbal number production: inferences from the performance of brain-damaged subjects. $f$ Exp Psychol: Gen 1986;115:307-30.

6 Damasio H, Damasio A. Lesion analysis in neuropsychology. New York. Oxford University Press, 1989.

7 De Renzi E, Faglioni P, Savoiardo M, Vignolo LA. The influence of aphasia and of hemispheric side of the cerebral lesion on abstract thinking. Cortex 1966;2:399-420.

8 Warrington EK. The fractionation of arithmetical skills: a single case study. Quart $f$ Exp Psychol 1982;34A:31-51.

9 Sokol SM, McCloskey M, Cohen NJ, Aliminosa D Cognitive representations and processes in arithmetic inferences fromthe performance of brain-damaged subjects. F Exp Psychol: Learn Mem and Cogn 1991;17: 355-76

10 Benton AL. Mathematical disability and the Gerstmann syndrome. In: Deloche $\mathrm{G}$ and Seron $\mathrm{X}$, eds. Mathematical disabilities $A$ cognitive neuropsychological perspective. Hillsdale: L Erlbaum, 1987:111-120

11 Bogousslavsky J, Assal G, Regli F. Infarctus du territoire de l'artere cérébrale antérieure gauche. II. Troubles du de l'artere cerébrale anterieure gauch

12 Bogousslavsky J, Regli F. Anterior cerebral artery territory infarction in the Lausanne Stroke Registry. Clinical and etiologic patterns. Arch Neurol 1990;47:144-150. 